\title{
The prevention of oxidative stress improve asthmatic inflammation
}

\author{
Ana Lúcia Zanatta ${ }^{1}$, Pedro Vicente Michelotto Junior ${ }^{2}$, Anita Nishiyama ${ }^{1}$ \\ ${ }^{1}$ Department of Physiology, Federal University of Paraná, Curitiba, Brazil \\ ${ }^{2}$ School of Agricultural Sciences and Veterinary Medicine, Pontifical Catholic University of Paraná, São José dos Pinhais, Brazil \\ Email: michelottojunior@gmail.com
}

Received 30 September 2012; revised 17 November 2012; accepted 27 November 2012

\begin{abstract}
Asthma is a disease characterized by airway chronic inflammation and bronchial hyperactivity, involving the imbalance of oxidative and antioxidative agents. There is an increased free radical generation and a decreased antioxidant enzyme activity, which correlate with the severity of the disease. The oxidative stress triggers specific physiopathological changes in the respiratory tract as a result of proinflammatory molecule formation, such as isoprostanes and PAFlike lipids. The synthesis of these mediators is dependent on the availability of lipid substrates, such as PUFAs, which are present in cell membranes. Therefore, lipid oxidation may have an important role in the perpetuation and amplification of the asthmatic inflammatory response. This article will make considerations about how oxidative stress contributes to asthma pathogenesis.
\end{abstract}

Keywords: Asthma; Oxidative Stress; Inflammation; Lipid Mediators

\section{INTRODUCTION}

Asthma is a chronic inflammatory disease associated with oxidative and antioxidative disequilibrium. High oxygen levels of the pulmonary environment render it susceptible to oxidative stress reactions. Products generated in this condition, such as reactive oxidative species (ROS) and reactive nitrogen species (RNS), are biomarkers for disease progression [1].

ROS can be formed in metabolic reactions, such as in the mitochondrial electron transport during respiration. Ozone and air particulates, including pollutants such as cigarette smoke, represent another source of ROS generation that affects airways [2]. Regarding the airway inflammatory process such as asthma and chronic obstructive pulmonary disease, there is an increased production of ROS by inflammatory cells activation [3]. Therefore, ROS production, irrespective of its origin, becomes an important focus for therapeutic intervention.

This review will discuss the involvement of oxidative stress on asthmatic inflammation, aiming to consider the following question: does the modification of pulmonary oxidative status could affect the pathological feature of asthma? The displayed question is relevant since oxidative stress significantly influences the pathogenesis of asthma. It makes one take into account the requirement of an antioxidant therapy in association to the traditional drug treatments.

\section{GENERATION OF LIPID MEDIATORS IN ENZYMATIC AND NON-ENZYMATIC PATHWAYS}

\subsection{Enzymatic Pathways}

The main lipid mediators of asthmatic inflammation produced from arachidonic acid are prostaglandins, leukotrienes, thromboxanes and prostacyclins. It is well known that the bioactive eicosanoids biosynthesis is precisely regulated by several enzymes. Phospholipase $\mathrm{A}_{2}$ hydrolyzes the $s n-2$ ester bond of membrane phospholipids, releasing arachidonate. This fatty acid is then metabolized by enzymatic systems as cyclooxygenases (COX) and lipoxygenases (LOX) that results in aforementioned eicosanoids formation [4].

Besides COX and LOX-derived substances, another lipid mediator that plays a central role in asthma is the Platelet Activating Factor (PAF). PAF synthesis is a highly regulated enzymatic pathway and usually involves a two-step mechanism [5]. There is an initial esterification of a lysophosphatidylcholine at the $s n-2$ glycerol skeleton position. Later, bioactive PAF is produced by acetyl transferase acetylation [6]. PAF is a biologically active phospholipid [6] and one of the most potent proinflammatory mediator identified to date [7]. The correlation of PAF and asthmatic pulmonary inflammation is pointed out by the demonstration that PAF level is increased in asthmatic patients and decreased after immunotherapy [7-9]. 


\subsection{Non-Enzymatic Pathways}

The oxidative stress observed in asthma makes the environment conducive to oxidative reactions producing omega-6/omega-3 polyunsaturated fatty acids (PUFAs)derived inflammatory mediators [10]. Thus, one may consider that non-enzymatic reactions or without regulation by antioxidant systems may be an important source of inflammatory mediators in asthma.

Lipoperoxidation occurs as a chain reaction initiated by free radicals formation on the fatty acid esterified to the phospholipid [11]. It propagates by reacting to an oxygen molecule and result in a lipid peroxyl radical, which is capable of abstracting hydrogen from another PUFA [12]. A great quantity of lipid hydroperoxides is produced, which are degraded in subsequent reactions [13]. Thus, various chemical species are formed, among them some species with proinflammatory activity [6], as isoprostanes and PAF-like molecules.

Isoprostanes. The isoprostanes are prostaglandin-like compounds formed from the free radical-catalyzed peroxidation of PUFAs, especially arachidonic acid, without the action of COX [1]. Their structural similarity to prostanoids (prostaglandins and thromboxanes) make them capable to activate thromboxane (receptor TP) [1, 13,14] and prostaglandins receptors (receptors FP, EP1-4) [1], explaining their biological activity [13,14]. Therefore, isoprostanes may exert many physiopathological effects, such as airway smooth muscle tonus regulation and hyperresponsiveness $[1,13]$, pulmonary vasoconstriction [14] and plasma exudation in alveolar microenvironment [13]. These effects are compatible with asthma features. Indeed, high levels of F2-isoprostanes have been observed in animal models of asthma and in asthmatic patients. Additionally, the severity of asthma is positively associated with the concentration of 8 -isoPGF2 $\alpha$ (8-iso-prostaglandin F2 $\alpha$ ) [13].

Isoprostanes modulate airway smooth muscle cell function by the regulation of the chemokines production. It was shown that they may promote the increase in GCSF-induced (granulocyte colony stimulating factor) IL$1 \beta$ in human airway smooth muscle cells [1]. This is a significant role of isoprostanes, since it represents an indirect pathway of inflammatory response amplification. Other important activities of isoprostanes in the inflammatory process are: stimulation of macrophage and neutrophil adhesion to endothelial cells, augmentation of macrophage IL-8 expression and increase of the vascular permeability [1]. Therefore, the complexity and multiplicity of isoprostanes actions may represent a focus of interest in the research for asthma management and new treatment therapies.

PAF-like. A PAF mimetics molecule with important proinflammatory effects generated non-enzymatically from phosphatidylcholine. Some decades ago, Smiley [15] carried out an experiment consisted of 1-palmitoyl-2-arachidonoyl-sn-glycero-3-phosphocholine fragmentation by an uncontrolled free radical-catalyzed reaction. This process generated several lipid fragments, which were capable of activating polymorphonuclear cells. The hydrolysis of the $s n-2$ residue of these fragments by PAF acetylhydrolase avoided their biological activity. In the same study, it was found that neutrophil activation is completely blocked by a specific antagonist of PAF receptor. Therefore, Smiley [15] evidenced that oxidation of phospholipids containing acyl residues (PUFA) at the $s n-2$ position generate molecules with PAF-like biological activity. In their study, Smiley [15] already suggested that PAF-like phospholipids may be generated under pathological conditions in which ROS play an essential role [15]. Currently, the participation of PAF-like molecules in many conditions is well established, such as in atherosclerosis and asthma $[8,16]$.

In spite of being less potent than PAF, PAF-like lipids are generated in high quantities and in an uncontrolled manner, rendering their action important in inflammation [17]. In asthma, the most important immediate activities of PAF include leukocyte chemotaxis and activation, increase of vascular permeability, vasoconstriction, bronchoconstriction and bronchial hyperresponsiveness [5,7, 8,18]. PAF also has long term effects [7] that determine the severity of asthma. It was demonstrated that in chronic inflammation, PAF induces airway vascular remodeling, caliciform cell hyperplasia and mucin gene expression [7].

\section{ASTHMA: A DISEASE ASSOCIATED WITH OXIDATIVE STRESS}

In asthma, the inflammatory response is accompanied by the generation of reactive oxygen and nitrogen species (ROS and RNS) [19-21], a process that is regulated by inflammatory mediators. Among the ROS and RNS are superoxide anion, hydrogen peroxide, hydroxyl radical, perchloric acid, nitric oxide (NO) and peroxynitrite [19, $20,22,23]$. ROS and RNS are produced by inflammatory cells, including eosinophils, neutrophils and macrophages. These oxidants participate in disease progression, especially during the exacerbation of asthmatic inflammation $[19,22]$. The combination of these facts, including increase in ROS and RNS, oxidative stress and non-enzymatic production of inflammatory mediators, give us indication of the oxidative imbalance importance in asthma.

Antioxidant defense. Another feature found in asthma is the loss of antioxidant defenses [19,20,22,24,25]. Lungs and blood have many enzymatic and non-enzymatic antioxidants, like glutathione peroxidase (GPx), superoxide 
dismutase (SOD), catalase (CAT), heme oxygenase, glutaredoxin, thioredoxin, peroxiredoxin, glutathione, carotenoids, vitamins $\mathrm{E}, \mathrm{A}$ and $\mathrm{C}$-all with the purpose of counteracting oxidants toxicity $[19,20,25]$. Increased ROS and RNS lead to structural and functional protein modification that are biologically relevant for inflammation initiation and maintenance [19]. Such alterations include the reduced activity of SOD, CAT and GPx [19,21-23,25]. Consequently, ROS and RNS are generated in large quantities in the lungs of asthmatic subjects that can overwhelm the antioxidant system activity of the organism. It leads to an uncontrolled production of new reactive species even more deleterious to the organism. In the absence of antioxidant defenses, the reactive species cause many forms of cell lesions, such as damage to DNA and to lipid membranes $[21,22,26]$.

Airway smooth muscle. Airway smooth muscle importance goes beyond its contractile function, since it can contribute to the chronic inflammatory process and participate in the remodeling of airways [3]. Its relevance derives from the fact that smooth muscle cells can express and produce cytokines and chemokines $[3,13]$, interact with extracellular matrix proteins and, in some circumstances, produce collagen [3]. In the context of oxidative stress, it can generate inflammatory mediators that interfere with parameters such as: smooth muscle contraction, airway hyperreactivity, mucus hypersecretion, epithelial desquamation and vascular exudation [13]. Therefore, the effects of oxidative stress on airway smooth muscle are significant in the chronic inflammatory process observed in asthma.

Intracellular signaling pathways. Under pathological conditions, intense ROS production by cells can cause changes in signal transduction and in gene expression [27]. Oxidative stress activates transcription factors, such as NF- $\mathrm{KB}$ and AP-1, and signaling pathways that culminate with the expression of several proinflammatory genes [3]. Some signaling pathways activated by ROS are: extracellular signal regulated kinases (ERKs), c-Jun $\mathrm{N}$-terminal kinases (JNKs), p38 MAP kinases (MAPKs), Protein Kinase C (PKC), Phosphatidylinositol 3-kinases (PI3K/Akt) and thyrosine kinase growth factor receptors [27]. Thus, ROS play an important physiopathological role as second messenger [27], with changes in inflammatory response.

In summary, in asthmatic inflammation there is an increased ROS and RNS production and a decreased antioxidant system activity, which has consequences in airway smooth muscles reactivity. These reactive species also act as markers of the asthma intensity and exacerbation, in addition to being correlated with disease severity. Its relevance as a biomarker is justified by the fact that they interfere with parameters that characterize the asthmatic inflammation.

\section{PERSPECTIVES}

As discussed above we conclude that oxidative stress can interfere directly in several aspects of asthmatic inflammation, increasing the severity of the pathology. If PUFAs are components of membrane phospholipids, the attack of these structures by reactive species can generate proinflammatory molecules, which are key regulators of allergic pulmonary inflammation. In this way, the manipulation of diets can have a key role in regulating the asthmatic condition by changing the availability of lipids susceptible to oxidation by those oxidative agents, and consequently generated proinflammatory molecules. Furthermore, oxidative stress may result not only of increased oxidative species, but also by a reduction in antioxidant activity. Therefore, the association of antioxidant therapy to the conventional treatment may be an alternative to improve patient response to therapy. Two considerations need to be made to give more consistency to our suggestion. 1) The lung has the peculiarity of the high flow oxygen which favors oxidation reactions and the generation of proinflammatory molecules. 2) Epidemiological data suggest that low vegetable and fruit intake are associated with the development of asthma [28]. Thus, antioxidant addition to the diet could protect against the noxious oxidant agents, as well as inhibiting subsequent steps of inflammatory response amplification, decreasing the severity and the perpetuation of asthmatic inflammation. Accordingly, the establishment of protocols of antioxidant therapy associated with conventional treatment may significantly improve the asthmatic patients quality life.

If oxidative stress affect asthma pathology as is stated in this review, a future perspective could guide studies in terms of new technologies for antioxidants distribution, as through stem cells technology and gene therapy on redox system.

\section{REFERENCES}

[1] Voynow, J.A. and Kummarapurugu, A. (2011) Isoprostanes and asthma. Biochimica et Biophysica Acta, 1810, 10911095. doi:10.1016/j.bbagen.2011.04.016

[2] Kim, B.J. and Hong, S.J. (2012) Ambient air pollution and allergic diseases in children. Korean Journal of Pediatrics, 55, 185-192.

[3] Chung, K.F. and Marwick, J.A. (2010) Molecular mechanisms of oxidative stress in airways and lungs with reference to asthma and chronic obstructive pulmonary disease. Annals of the New York Academy of Sciences, 1203, 85-91. doi:10.1111/j.1749-6632.2010.05600.x

[4] Astudillo, A.M., Perez-Chacon, G., Balboa, M.A. and Balsinde, J. (2009) Arachidonic acid mobilization by stimuli of the innate immune response. Inmunología, 28, 182192. doi:10.1016/S0213-9626(09)70041-1 
[5] Zimmerman, G.A., McIntyre, T.M., Prescott, S.M. and Stafforini, D.M. (2002) The platelet-activating factor signaling system and its regulators in syndromes of inflammation and thrombosis. Critical Care Medicine, 30, S294S301. doi:10.1097/00003246-200205001-00020

[6] Marathe, G.K., Prescott, S.M., Zimmerman, G.A. and McIntyre, T.M. (2001) Oxidized LDL contains inflammatory PAF-like phospholipids. Trends in Cardiovascular Medicine, 11, 139-142. doi:10.1016/S1050-1738(01)00100-1

[7] Uhlig, S., Goggel, R. and Engel, S. (2005) Mechanisms of platelet-activating factor (PAF)-mediated responses in the lung. Pharmacological Reports, 57, 206-221.

[8] Muehlmann, L.A., et al. (2010) Dietary supplementation with soybean lecithin increases pulmonary PAF bioactivity in asthmatic rats. The Journal of Nutritional Biochemistry, 21, 532-537. doi:10.1016/j.jnutbio.2009.03.001

[9] Hsieh, K.H. and Ng, C.K. (1993) Increased plasma platelet-activating factor in children with acute asthmatic attacks and decreased in vivo and in vitro production of platelet-activating factor after immunotherapy. Journal of Allergy and Clinical Immunology, 91, 650-657. doi:10.1016/0091-6749(93)90271-G

[10] Marathe, G.K., et al. (2000) Bioactive phospholipid oxidation products. Free Radical Biology \& Medicine, 28, 1762-1770. doi:10.1016/S0891-5849(00)00234-3

[11] Cracowski, J.L., Durand, T. and Bessard, G. (2002) Isoprostanes as a biomarker of lipid peroxidation in humans: Physiology, pharmacology and clinical implications. Journal of Pharmacological Sciences, 23, 360-366.

[12] Frankel, E.N. (1984) Lipid oxidation-Mechanisms, products and biological significance. Journal of the American Oil Chemists Society, 61, 1908-1917. doi:10.1007/BF02540830

[13] Wood, L.G., Gibson, P.G. and Garg, M.L. (2003) Biomarkers of lipid peroxidation, airway inflammation and asthma. European Respiratory Journal, 21, 177-186. doi:10.1183/09031936.03.00017003a

[14] Basu, S. (2010) Bioactive eicosanoids: Role of prostaglandin $\mathrm{F}$ (2alpha) and $\mathrm{F}(2)$-isoprostanes in inflammation and oxidative stress related pathology. Molecules and Cells, 30, 383-391. doi:10.1007/s10059-010-0157-1

[15] Smiley, P.L., Stremler, K.E., Prescott, S.M., Zimmerman, G.A. and McIntyre, T.M. (1991) Oxidatively fragmented phosphatidylcholines activate human neutrophils through the receptor for platelet-activating factor. The Journal of Biological Chemistry, 266, 11104-11110.

[16] Pégorier, S., Stengel, D., Durand, H., Croset, M. and Ninio, E. (2006) Oxidized phospholipid: POVPC binds to platelet-activating-factor receptor on human macrophages Implications in atherosclerosis. Atherosclerosis, 188, 433443. doi:10.1016/j.atherosclerosis.2005.11.015
[17] Marathe, G.K., et al. (1999) Inflammatory platelet-activating factor-like phospholipids in oxidized low density lipoproteins are fragmented alkyl phosphatidylcholines. The Journal of Biological Chemistry, 274, 28395-28404. doi:10.1074/jbc.274.40.28395

[18] Prescott, S.M., Zimmerman, G.A. Stafforini, D.M. and Thomas, M.M. (2000) Platelet-activating factor and related lipid mediators. Annual Review of Biochemistry, 69, 419-445. doi:10.1146/annurev.biochem.69.1.419

[19] Comhair, S.A. and Erzurum, S.C. (2010) Redox control of asthma: Molecular mechanisms and therapeutic opportunities. Antioxidants \& Redox Signaling, 12, 93-124. doi:10.1089/ars.2008.2425

[20] Nadeem, A., Chhabra, S.K., Masood, A. and Raj, H.G. (2003) Increased oxidative stress and altered levels of antioxidants in asthma. Journal of Allergy and Clinical Immunology, 111, 72-78. doi:10.1067/mai.2003.17

[21] Reynaert, N.L., et al. (2007) Catalase overexpression fails to attenuate allergic airways disease in the mouse. The Journal of Immunology, 178, 3814-3821.

[22] Owayed, A., Dhaunsi, G.S. and Al-Mukhaizeem, F. (2008) Nitric oxide-mediated activation of NADPH oxidase by salbutamol during acute asthma in children. Cell Biochemistry and Function, 26, 603-608. doi:10.1002/cbf.1484

[23] Perišić, T., Sreckovic, M. and Matic, G. (2007) An imbalance in antioxidant enzymes and stress proteins in childhood asthma. Clinical Biochemistry, 40, 1168-1171. doi:10.1016/j.clinbiochem.2007.06.006

[24] Kaleli, S., Akkaya, A., Akdogan, M. and Gültekin, F. (2006) The effects of different treatments on prolidase and antioxidant enzyme activities in patients with bronchial asthma. Environmental Toxicology and Pharmacology, 22, 35-39. doi:10.1016/j.etap.2005.11.001

[25] Mak, J.C.W., et al. (2004) Systemic oxidative and antioxidative status in Chinese patients with asthma. Journal of Allergy and Clinical Immunology, 114, 260-264. doi:10.1016/j.jaci.2004.05.013

[26] Zimmerman, G.A., Prescott, S.M. and McIntyre, T.M. (1995) Oxidatively fragmented phospholipids as inflammatory mediators: The dark side of polyunsaturated lipids. Journal of Nutrition, 125, 1661S-1665S.

[27] Lee, I.T. and Yang, C.M. (2012) Role of NADPH oxidase/ROS in pro-inflammatory mediators-induced airway and pulmonary diseases. Biochemical Pharmacology, 84, 581-590. doi:10.1016/j.bcp.2012.05.005

[28] Nurmatov, U., Devereux, G. and Sheikh, A. (2011) Nutrients and foods for the primary prevention of asthma and allergy: Systematic review and meta-analysis. Journal of Allergy and Clinical Immunology, 127, 724-733. doi:10.1016/j.jaci.2010.11.001 